\title{
Polymorphism of the FTO Gene Influences Body Weight in Children with Type 1 Diabetes without Severe Obesity
}

\author{
Włodzimierz Luczyński, ${ }^{1}$ Wojciech Fendler, ${ }^{2}$ Anna Ramatowska, ${ }^{3}$ Agnieszka Szypowska, \\ Agnieszka Szadkowska, ${ }^{2}$ Wojciech Młynarski, ${ }^{2}$ Miron Chumiecki, ${ }^{4}$ \\ Przemysława Jarosz-Chobot, ${ }^{4}$ Joanna Chrzanowska, ${ }^{5}$ Anna Noczyńska, ${ }^{5}$ \\ Agnieszka Brandt, ${ }^{6}$ Małgorzata Myśliwiec, ${ }^{6}$ Barbara Głowińska-Olszewska, ${ }^{1}$ \\ Pawel Bernatowicz, ${ }^{7}$ Oksana Kowalczuk, ${ }^{8}$ and Artur Bossowski ${ }^{1}$ \\ ${ }^{1}$ Department of Pediatrics, Endocrinology, Diabetology with Cardiology Division, Medical University of Bialystok, \\ Bialystok 15-274, Poland \\ ${ }^{2}$ Department of Paediatrics, Oncology, Haematology and Diabetology, Medical University of Lodz, Lodz 91-738, Poland \\ ${ }^{3}$ Department of Paediatrics, Medical University of Warsaw, Warsaw 01-184, Poland \\ ${ }^{4}$ Department of Paediatrics, Paediatric Endocrinology and Diabetology, Medical University of Silesia, in Katowice, \\ Katowice 40-752, Poland \\ ${ }^{5}$ Department of Endocrinology and Diabetology of Children and Adolescents, Wroclaw Medical University, Wroclaw 50-268, Poland \\ ${ }^{6}$ Department of Paediatrics, Diabetology and Endocrinology, Medical University of Gdansk, Gdansk 80-211, Poland \\ ${ }^{7}$ Department of Haematology, Medical University of Bialystok, Bialystok 15-274, Poland \\ ${ }^{8}$ Department of Clinical Molecular Biology, Medical University of Bialystok, Bialystok 15-274, Poland
}

Correspondence should be addressed to Włodzimierz Łuczyński; w.luczynski@wp.pl

Received 13 May 2014; Accepted 22 July 2014; Published 19 August 2014

Academic Editor: Lorenzo Piemonti

Copyright (C) 2014 Włodzimierz Łuczyński et al. This is an open access article distributed under the Creative Commons Attribution License, which permits unrestricted use, distribution, and reproduction in any medium, provided the original work is properly cited.

\begin{abstract}
The objective was to compare the impact of clinical and genetic factors on body mass index (BMI) in children with type 1 diabetes (T1DM) without severe obesity. A total of 1,119 children with T1DM (aged 4-18 years) were qualified to take part in the study. All children were genotyped for variants of FTO, MC4R, INSIG2, FASN, NPC1, PTER, SIRT1, MAF, IRT1, and CD36. Results. Variants of FTO showed significant association with BMI-SDS in the T1DM group. The main factors influencing BMI-SDS in children with T1DM included female gender $(P=0.0003)$, poor metabolic control $(P=0.0001)$, and carriage of the A allele of the FTO rs9939609 gene $(P=0.02)$. Conclusion. Our research indicates, when assessing, the risk of overweight and obesity carriage of the A allele in the rs 9939609 site of the FTO gene adds to that of female gender and poor metabolic control. This trial is registered with ClinicalTrials.gov (NCT01279161).
\end{abstract}

\section{Introduction}

The incidence of overweight and obesity among children has greatly increased in the past few decades [1]. Our stable genetic system, favouring the accumulation of energy, has become a problem in times of excessive access to food and low physical activity. Recently, promising data has been found regarding polymorphisms of FTO, MC4R, INSIG2, CD36 genes, and many others [2]. The influence of FTO and MC4R genes on body mass is mediated primarily through food consumption (appetite regulation) rather than through a decrease in activity-related energy expenditure (review [3]). Controversial association results for INSIG2 on body mass index may be explained by interactions with age and with MC4R [4].

The intensive insulin therapy for type 1 diabetes (T1DM) is associated with weight gain, abdominal obesity, dyslipidaemia, hypertension, and the presence of features of atherosclerosis development in imaging [5]. For unknown reasons, sex differences are observed in this field: in contrast to the nondiabetic population, girls and women with diabetes 
are characterized by a greater number of risk factors for cardiovascular diseases than boys and young men [6]. Since the association of known genetic polymorphisms with body weight is not very strong, the objective of this study was to compare the impact of clinical and genetic factors on body mass index in a large cohort of not severely obese children with T1DM.

\section{Materials and Methods}

2.1. Patients and Methods. A total of 1,119 children with T1DM from study centers of the PolPeDiab research group were qualified to take part in the study. These six centers have treated $60 \%$ of children with diabetes in Poland (10$90 \%$ of patients from each center). The study inclusion criteria were Polish ethnicity, age 4-18 years, diagnosis of T1DM (according to ISPAD), treatment with insulin alone for at least one year using the same intensive regimen with the use of pens or personal insulin pumps, and end of remission (based on the definition suggested by Mortensen et al.: $\mathrm{HbAlc} \%+(4 \times$ insulin dose $\mathrm{U} / \mathrm{kg} /$ day $)>9$ [7] $)$. Psychiatric abnormalities (including nutritional disorders), evidence of chromosomal disorders in physical examination, and hormonal and autoimmune disorders (celiac disease, diseases of the thyroid, adrenals, and gonads) were excluded $(20 \%)$. Children with severe obesity (SDS-BMI > 3) were also excluded from the study (4\% of all patients with T1DM). Subjects did not take any medications apart from insulin. The study was conducted between October 2009 and December 2011. The study design was approved by the Ethics Committee at the Medical University of Bialystok (no. R-I002/347/2009). The parents/guardians of each subject gave their written, informed consent for study participation.

\subsection{Anthropometric Methods, Additional Assessments, and} Definitions. Subject examination included measurements of height ( $\mathrm{cm}$, Harpenter's stage-meter) and weight $(\mathrm{kg})$ conducted by trained members of the study team. Height and weight were measured in light sports garments or underwear, without shoes, with an empty bladder. Each measurement was taken twice. Height was measured with a precision of $0.1 \mathrm{~cm}$. With the difference in height measurements of $>0.5 \mathrm{~cm}$ a third measurement was taken. Weight was measured with a precision of $0.1 \mathrm{~kg}$; with the results divergent by $>0.3 \mathrm{~kg}$, a third measurement was taken. The BMI $\left(\mathrm{kg} / \mathrm{m}^{2}\right)$ and BMI-SDS values were calculated [8]. The intra- and interobserver agreements were calculated according to the statistical methods proposed by Bland and Altman [9]. The intra- and interobserver coefficient of variation was $1.0 \%$ and $1.5 \%$ (resp.) for the height and $0.8 \%$ and $1.1 \%$ for weight. The collected data were referenced against the recently updated norms for Polish children, including weight centile charts according to sex and age (Polish nationwide OLAF project) [8].

The daily insulin dosage was acknowledged to be the mean dosage from the 3 days prior to the current visit (in the case of pens, the data was obtained from blood glucose selfmonitoring diaries, and in the case of insulin pumps the data was obtained from device software). Study subjects were on a diabetic diet, they calculated carbohydrate and protein-fat equivalents (pumps), and all had been educated on functional insulin therapy.

Glycosylated hemoglobin (HbAlc) rates were evaluated with the use of high-performance liquid chromatography (HPLC) in the reference laboratories. HbAlc values obtained from the laboratories were mathematically standardized to the Diabetes Control and Complications Trial normal range. Mean $\mathrm{HbAlc}$ for the previous year of treatment (minimum 3 measurements) were calculated. Only children with all data available were qualified for analysis.

2.3. Genotyping. All children were assessed for the following polymorphisms: FTO (rs9939609: A/T, rs1421085: C/T, rs17817449: G/T, rs1121980: A/G), MC4R (rs17782313: C/T), INSIG2 (rs7566605: C/G), FASN (rs2229422: A/G), NPC1 (rs1805081: C/T), PTER (rs10508503: C/T), SIRT1 (rs7895833: A/G, rs1467568: A/G), MAF (rs1424233: C/T), and CD36 (rs3211867: A/C, rs3211883: A/T, rs1527483: A/G) via allelic discrimination with ABI 7900HT Fast Real-Time PCR System with SDS 2.1 software (Applied Biosystems, Foster City, CA, USA). During blood sampling for required laboratory diagnostic tests, a volume of $0.5 \mathrm{~mL}$ of blood was collected for genotyping. All children were assessed for the polymorphisms via allelic discrimination with ABI 7900HT Fast Real-Time PCR System with SDS 2.1 software (Applied Biosystems, Foster City, CA, USA). The genetic variants were evaluated with the use of validated commercially available probes included in the TaqMan SNP Genotyping Assays (Applied Biosystem). DNA was isolated from venous whole blood with TaqMan Sample-to-SNP Kit (Applied Biosystems) according to the manufacturer's instructions. The assay was conducted on 384-well plates in $5 \mu \mathrm{L}$; the mixture contained $1 \mu \mathrm{L}$ of DNA, $2.5 \mu \mathrm{L}$ TaqMan GTXpress Master Mix (Applied Biosystems), $0.25 \mu \mathrm{L}$ TaqMan Genotyping Assay Mix 20x (Applied Biosystems), and $1.25 \mu \mathrm{L}$ DNase-free water. The temperature profile of the reaction was as follows: initial denaturation at $95^{\circ} \mathrm{C}$ for $10 \mathrm{~min}, 40$ consecutive cycles of denaturation at $92^{\circ} \mathrm{C}$ for $15 \mathrm{~s}$, and hybridization/elongation at $60^{\circ} \mathrm{C}$ for $60 \mathrm{~s}$. As contamination control, each reaction plate contained negative assays containing water instead of DNA. A total of $99.3 \%$ correct reactions were recorded.

2.4. Statistical Analysis. Univariate analysis was performed using the Student's $t$-test in the case of continuous variables and the Chi-square test for nominal ones. Frequencies of observed alleles were tested against the Hardy-Weinberg equilibrium using the Chi-square test. As the study was not aimed at establishing individual effects of polymorphisms on the risk of diabetes, we did not perform multivariate association analysis. Multivariate linear regression was used to evaluate the impact of all polymorphic variants and clinical features on BMI-SDS. Second-order interactions were also analysed to evaluate any potential interactions of the presence of diabetes and genotypes at particular loci. The study was planned to provide $80 \%$ statistical power to detect differences greater than 0.2 of BMI-SDS with type 1 error probability 


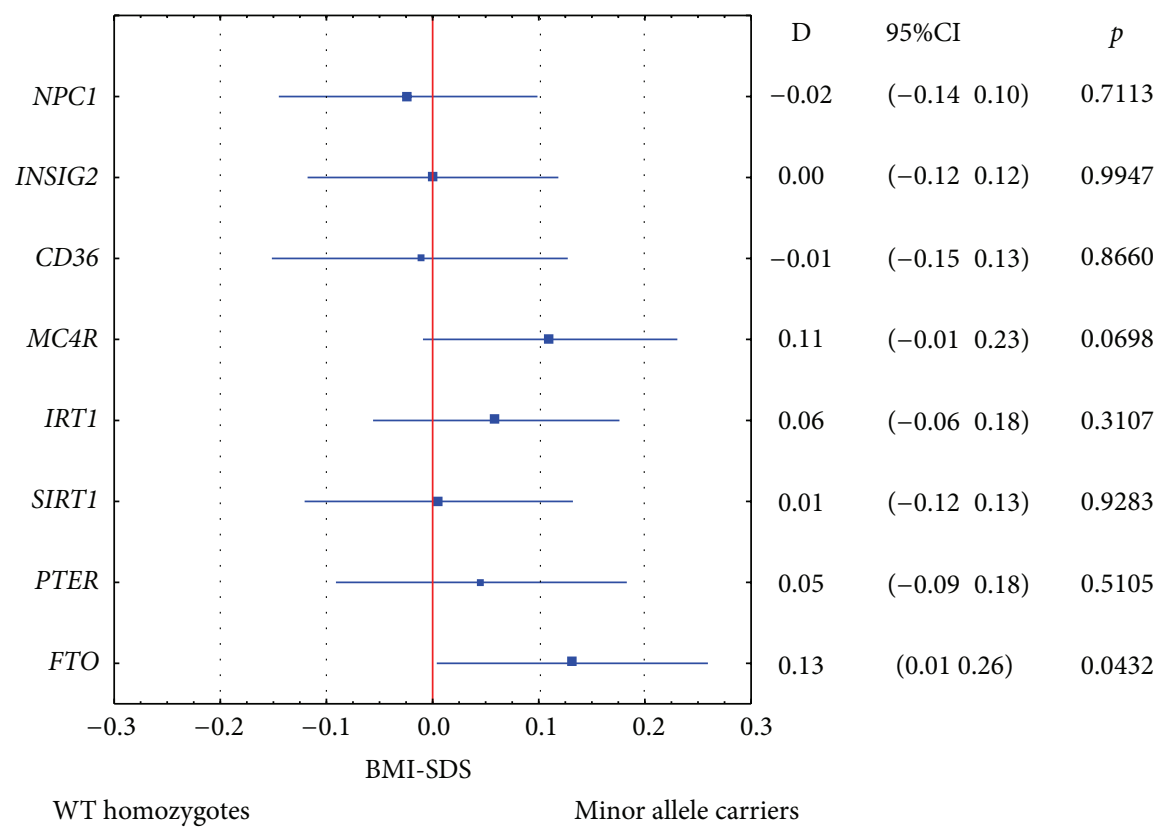

FIGURE 1: The influence of tested polymorphic variants on standardized BMI (BMI-SDS) in children with type 1 diabetes. D: difference of means, 95\% CI: 95\% confidence interval, and WT: wild-type.

TABLE 1: Clinical data of children with diabetes.

\begin{tabular}{lcc}
\hline & Mean & T1DM \\
\hline Age & 13.39 & SD \\
Height-SDS & 0.22 & 3.42 \\
Weight-SDS & 0.50 & 1.25 \\
SDS-BMI & 0.42 & 1.06 \\
HbAlc & 7.77 & 0.94 \\
Insulin dose (U/kg/day) & 0.79 & 1.52 \\
\hline
\end{tabular}

lower than 0.15 , for heterozygosity of the tested SNPs equal to 0.5 . Since there were 9 SNPs in question, actual power for respective comparisons ranged between 70 and $90 \%$.

\section{Results}

3.1. Study Group Characteristics. Baseline characteristics of the studied group are presented in Table 1. In the group of children with type 1 diabetes, the girls were characterized by higher values of standardized BMI compared to boys $(0.52 \pm 0.94$ versus $0.31 \pm 0.91, P=0.0001)$. In terms of other parameters, there were no gender differences. BMI-SDS did not correlate strongly or significantly with age, duration of diabetes, insulin dose, or HbAlc levels ( $r$ from -0.007 to 0.093, all $P$ values $>0.05$ )

3.2. Results of the Genetic Studies. The distribution of alleles at two of the studied loci (FASN and MAF) deviated from the ones expected in the Hardy-Weinberg equilibrium, which excluded them from further analysis of polymorphic allele carriage effects and multivariate models.

Of all tested SNPs only FTO showed any significant association with BMI-SDS in the T1DM group (Figure 1). Recessive models of SNP effects were tested, but, apart from FTO, which was significant for both recessive and dominant modes of action, none of the analysed polymorphisms showed differences even of borderline significance $(P>0.1$ in all cases).

Carriers of FTO polymorphic allele had shown higher BMI-SDS scores regardless of the patient's gender (Table 2). The impact of the patient's age and other genetic factors remained nonsignificant $(P>0.15)$. Except for female gender and carriage of the polymorphic allele of FTO, the third significant factor associated with higher BMI-SDS was confirmed to be HbAlc level (Table 2).

\section{Discussion}

Our study of the group of individuals without severe obesity indicates an effect of FTO gene polymorphism on BMI in children with T1DM. Moreover, gender and metabolic control significantly influenced standardized BMI.

Previously we have shown an association of FTO with body weight in Polish healthy children, similar to other authors [10, 11]. Nevertheless, it seems that there is no correlation between the genotype predisposing to obesity and the risk of autoimmune reactions and the formation of T1DM [12]. On the other hand, overweight did not predispose to more rapid progression of autoimmune response in patients enrolled in the study BABYDIAB [13]. According to the data of other authors, the influence of the genetic polymorphisms we studied on body weight is not strong, and severe obesity is 
TABLE 2: Multivariate regression model including the clinical and genetic features influencing the standardized body mass index in children with type 1 diabetes.

\begin{tabular}{lccr}
\hline $\begin{array}{l}\text { Factors affecting BMI among } \\
\text { children with diabetes }\end{array}$ & Regression coefficient & Partial correlation coefficient & \\
\hline Intercept & -0.201654 & 0.119130 & 0.205718 \\
Female sex & 0.110525 & 0.074232 & 0.000337 \\
FTO polymorphic allele carriage & 0.075598 & 0.124044 & 0.024979 \\
Mean yearly HbAlc level & 0.075028 & 0.000190 \\
\hline
\end{tabular}

due to other causes. Therefore, we excluded from the current analysis children with a value of standardized BMI above 3. However, our research indicates, when assessing, the risk of overweight and obesity carriage of the A allele in the FTO variant rs9939609 should be taken into account. The impact of this polymorphism on body weight in patients with T1DM was also observed in a study of the genetics of kidney diseases in diabetes [14]. The most interesting result of our observations is the fact that FTO variants have an additional effect, besides clinical features, on the body weight in diabetic children.

The FTO, INSIG2, CD36, and MC4R genes tested in our study are the main polymorphisms associated with body weight [15]. Substantial problems concerning obesity and T1DM include difficulty in distinguishing the types of disease in adolescents with features of insulin resistance, weight gain during intensive insulin therapy, the potential impact of obesity on long-term complications, and the relationship of body weight with metabolic control. In a large group of German and Austrian children with type 1 diabetes a higher incidence of risk factors for cardiovascular disease in girls compared to boys (metabolic control, body mass index, total cholesterol, triglycerides, and blood pressure) was observed, except for abnormal HDL-cholesterol and cigarette smoking [16]. The reasons for poor metabolic control and obesity in adolescent girls with type 1 diabetes remain unknown. Obviously, patients with obesity, poor self-control, and poor metabolic control constitute a risk group for eating disorders [17].

Recently, a significant association between the body weight of adolescents with type 1 diabetes and their parents' body weight was demonstrated [18]. Interestingly, metabolic control correlated not only with the patients' body weight, but also with that of their parents. It is therefore clear that obesity is a familial problem for children with T1DM. It remains an open question whether, in these cases, obesity has an environmental background (the same style of life) or genetic background.

\section{Conclusions}

The main factors influencing body weight in children with diabetes include female gender, poor metabolic control, and carriage of the A allele of the FTO rs9939609 variant. Improvement of metabolic control of T1DM is the major modifiable factor associated with BMI and should be considered of crucial importance, particularly in girls T1DM and high BMI.

\section{Conflict of Interests}

The authors declare that there is no conflict of interests regarding the publication of this paper.

\section{Acknowledgment}

The authors have made this work on behalf of the PolPeDiab group.

\section{References}

[1] M. Chrzanowska, S. Koziel, and S. J. Ulijaszek, "Changes in BMI and the prevalence of overweight and obesity in children and adolescents in Cracow, Poland, 1971-2000," Economics \& Human Biology, vol. 5, no. 3, pp. 370-378, 2007.

[2] T. M. Frayling, N. J. Timpson, M. N. Weedon et al., "A common variant in the FTO gene is associated with body mass index and predisposes to childhood and adult obesity," Science, vol. 316, no. 5826, pp. 889-894, 2007.

[3] J. Cecil, M. Dalton, G. Finlayson, J. Blundell, M. Hetherington, and C. Palmer, "Obesity and eating behaviour in children and adolescents: contribution of common gene polymorphisms," International Review of Psychiatry, vol. 24, no. 3, pp. 200-210, 2012.

[4] D. Malzahn, M. Müller-Nurasyid, I. M. Heid, H. E. Wichmann, The KORA Study Group6, and H. Bickeböller, "Controversial association results for INSIG2 on body mass index may be explained by interactions with age and with MC4R," European Journal of Human Genetics, 2014.

[5] C. Steigleder-Schweiger, B. Rami-Merhar, T. Waldhör et al., "Prevalence of cardiovascular risk factors in children and adolescents with type 1 diabetes in Austria," European Journal of Pediatrics, vol. 171, no. 8, pp. 1193-1202, 2012.

[6] S. Krishnan, D. A. Fields, K. C. Copeland, P. R. Blackett, M. P. Anderson, and A. W. Gardner, "Sex differences in cardiovascular disease risk in adolescents with type 1 diabetes," Gender Medicine, vol. 9, no. 4, pp. 251-258, 2012.

[7] H. B. Mortensen, P. Hougaard, P. Swift et al., "New definition for the partial remission period in children and adolescents with type 1 diabetes," Diabetes Care, vol. 32, no. 8, pp. 1384-1390, 2009.

[8] Z. Kułaga, M. Litwin, M. Tkaczyk et al., "Polish 2010 growth references for school-aged children and adolescents," European Journal of Pediatrics, vol. 170, no. 5, pp. 599-609, 2011.

[9] J. M. Bland and D. G. Altman, "Statistical methods for assessing agreement between two methods of clinical measurement," The Lancet, vol. 1, no. 8476, pp. 307-310, 1986.

[10] B. Pyrzak, A. Wisniewska, A. Majcher, A. Tysarowski, and U. Demkow, "Relation of fat-mass and obesity-associated gene 
polymorphism to fat mass content and body mass index in obese children," Advances in Experimental Medicine and Biology, vol. 756, pp. 255-262, 2013.

[11] W. Luczynski, G. Zalewski, and A. Bossowski, "The association of the FTO rs9939609 polymorphism with obesity and metabolic risk factors for cardiovascular diseases in polish children," Journal of Physiology and Pharmacology, vol. 63, no. 3, pp. 241-248, 2012.

[12] S. F. Field, J. M. M. Howson, N. M. Walker, D. B. Dunger, and J. A. Todd, "Analysis of the obesity gene FTO in 14,803 type 1 diabetes cases and controls," Diabetologia, vol. 50, no. 10, pp. 2218-2220, 2007.

[13] C. Winkler, J. Raab, H. Grallert, and A. Ziegler, "Lack of association of type 2 diabetes susceptibility genotypes and body weight on the development of islet autoimmunity and type 1 diabetes," PloS ONE, vol. 7, no. 4, Article ID e35410, 2012.

[14] H. F. Gu, A. Alvarsson, and K. Brismar, "The common FTO genetic polymorphism rs9939609 is associated with increased BMI in type 1 diabetes but not with diabetic nephropathy," Biomarker Insights, vol. 5, pp. 29-32, 2010.

[15] J. Zhao, J. P. Bradfield, M. Li et al., "The role of obesity-associated loci identified in genome-wide association studies in the determination of pediatric BMI," Obesity, vol. 17, no. 12, pp. 2254-2257, 2009.

[16] K. O. Schwab, J. Doerfer, W. Marg, E. Schober, and R. W. Holl, "Characterization of 33488 children and adolescents with type 1 diabetes based on the gender-specific increase of cardiovascular risk factors," Pediatric Diabetes, vol. 11, no. 5, pp. 357-363, 2010.

[17] T. R. Nansel, J. Tse, D. L. Haynie, S. N. Mehta, and L. M. B. Laffel, "Disordered eating behaviors are associated with poorer diet quality in adolescents with type 1 diabetes," Journal of the Academy of Nutrition and Dietetics, vol. 112, no. 11, pp. 1810-1814, 2012.

[18] A. L. P. Sands, L. A. Higgins, S. N. Mehta, T. R. Nansel, L. M. Lipsky, and L. M. B. Laffel, "Associations of youth and parent weight status with reported versus predicted daily energy intake and hemoglobin Alc in youth with type 1 diabetes mellitus," Journal of Diabetes Science and Technology, vol. 7, no. 1, pp. 263270, 2013. 


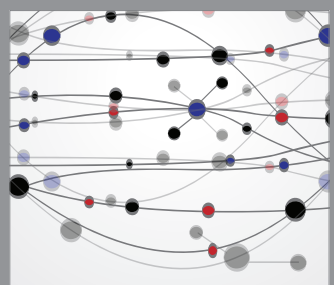

The Scientific World Journal
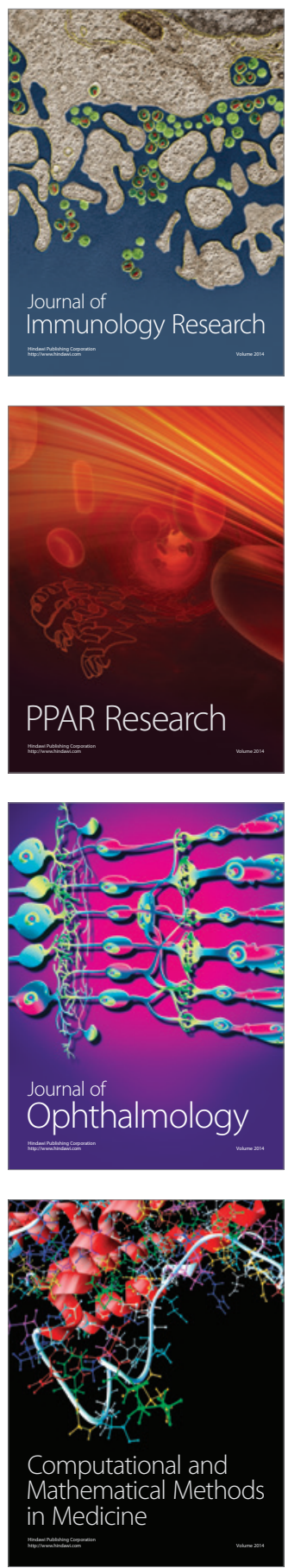

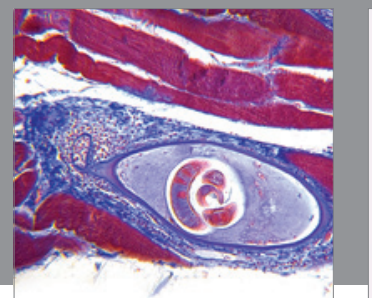

Gastroenterology

Research and Practice
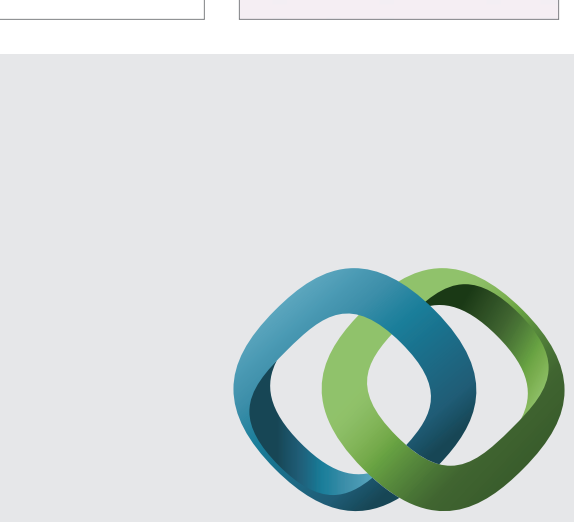

\section{Hindawi}

Submit your manuscripts at

http://www.hindawi.com
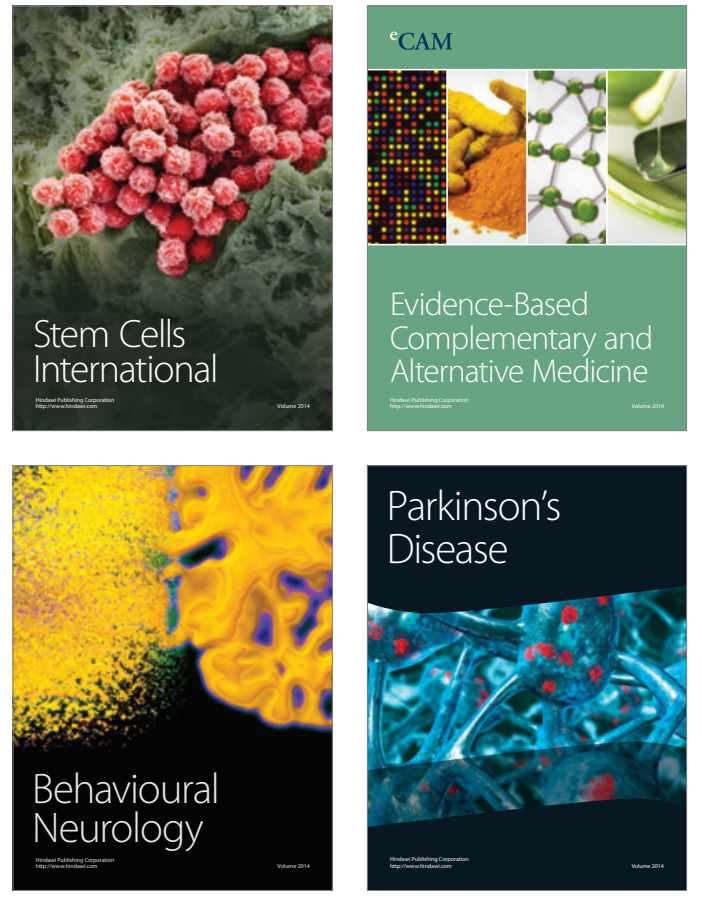
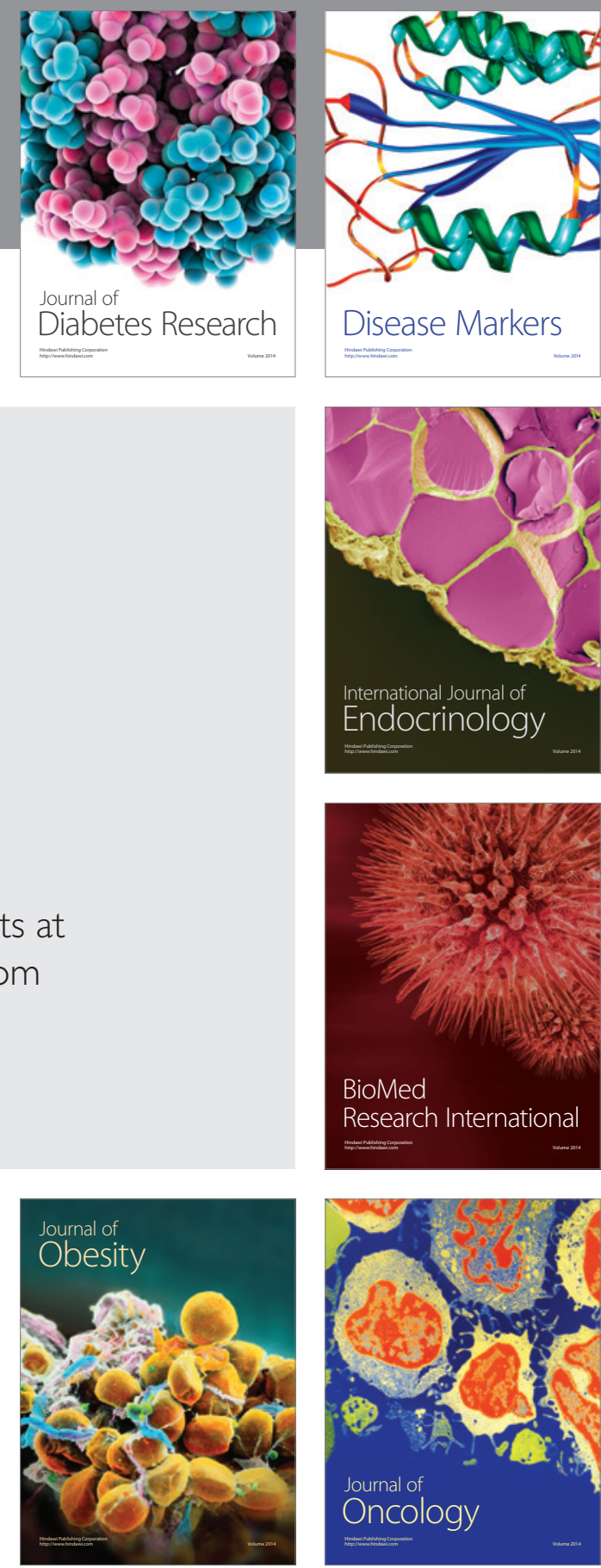

Disease Markers
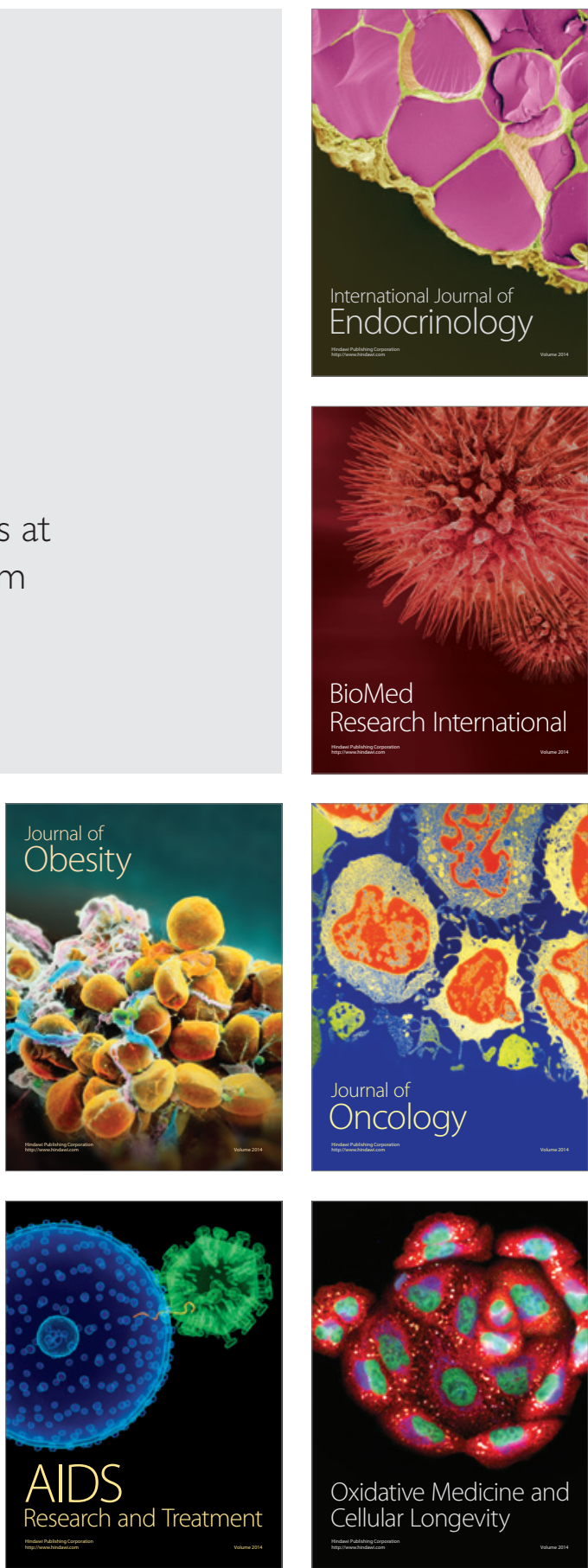